\title{
Nonequilibrium dynamical cluster approximation study of the Falicov-Kimball model
}

\author{
Andreas J. Herrmann, ${ }^{1, *}$ Naoto Tsuji, ${ }^{2}$ Martin Eckstein, ${ }^{3}$ and Philipp Werner ${ }^{1}$ \\ ${ }^{1}$ Department of Physics, University of Fribourg, 1700 Fribourg, Switzerland \\ ${ }^{2}$ RIKEN Center for Emergent Matter Science (CEMS), Wako 351-0198, Japan \\ ${ }^{3}$ Max Planck Research Department for Structural Dynamics, University of Hamburg-CFEL, 22761 Hamburg, Germany
}

(Received 16 September 2016; published 6 December 2016)

\begin{abstract}
We use a nonequilibrium implementation of the dynamical cluster approximation (DCA) to study the effect of short-range correlations on the dynamics of the two-dimensional Falicov-Kimball model after an interaction quench. As in the case of single-site dynamical mean-field theory, thermalization is absent in DCA simulations, and for quenches across the metal-insulator boundary, nearest-neighbor charge correlations in the nonthermal steady state are found to be larger than in the thermal state with identical energy. We investigate to what extent it is possible to define an effective temperature of the trapped state after a quench. Based on the ratio between the lesser and retarded Green's function, we conclude that a roughly thermal distribution is reached within the energy intervals corresponding to the momentum-patch dependent subbands of the spectral function. The effectively different chemical potentials of these distributions, however, lead to a very hot, or even negative, effective temperature in the energy intervals between these subbands.
\end{abstract}

DOI: 10.1103/PhysRevB.94.245114

\section{INTRODUCTION}

The nonequilibrium dynamics of correlated fermionic lattice systems is of interest in connection with pump-probe experiments on solids, experiments on ultracold atoms in an optical potential, and in the context of theoretical research on thermalization in many-body quantum systems. Over the past few years, the nonequilibrium extension of dynamical mean field theory (DMFT) [1,2] has been developed into a powerful approach that allows us to study the time evolution of high-dimensional lattice models. Applications of this method to the infinite-dimensional Hubbard model have produced interesting new insights, including, among others, the transient trapping of the system in prethermalized states [3] after an interaction quench [4], the existence of dynamical phase transitions [5], the appearance of nonthermal critical points [6-8] and nonthermal order [9] in antiferromagnetic systems, as well as first-order dynamical transitions in the Loschmidt echo $[10,11]$.

While local time-dependent fluctuations can be accurately described within DMFT, the spatial degrees of freedom are treated at the mean-field level. In low-dimensional systems, the effect of spatial fluctuations can be important for the dynamics, and to capture them, cluster extensions of nonequilibrium DMFT have been implemented. The one- and two-dimensional Hubbard model has been studied within the dynamical cluster approximation (DCA) in Ref. [12] using weak-coupling perturbation theory to solve the DMFT equations. In Ref. [13], a four-site DCA calculation was used to simulate the effect of short-range antiferromagnetic correlations on the dynamics of a photodoped Mott insulator. The relaxation rate of the photoexcited carriers was found to scale quadratically with the nearest-neighbor spin correlations. In the latter study, the DCA equations were solved using a self-consistent strong-coupling perturbation theory (non-crossing approximation) [4,14]. At the moment, technical limitations prevent an extension of

\footnotetext{
*andreas.herrmann@unifr.ch
}

these methods to the intermediate-coupling regime, where higher-order versions of the strong-coupling expansion have to be used. Unbiased numerical methods, such as quantum Monte Carlo [9,15] or DMRG [16,17], are severely limited by an exponential scaling of the computational effort with the accessible time range, and with cluster size. Hence, nonequilibrium DCA simulations of the Hubbard model are currently not only limited by the cluster size, which is essentially a memory issue, but most severely by the approximate methods used to solve the cluster impurity problem.

In this study, we explore the effect of short-range correlations in the Falicov-Kimball (FK) model [18], which admits an exact solution within DMFT and DCA. While the dynamics of the FK model differs in many respects from that of the Hubbard model, due to the immobility of one spin-species, it exhibits a rich phase diagram in equilibrium, with metallic, Mott-insulating, and also long-range-ordered phases [19]. Hence, it is interesting to explore the effect of intersite correlations on the relaxation properties of this model.

It is known from single-site nonequilibrium DMFT studies that the FK model does not thermalize after an interaction quench [20], because on the one hand the distribution of immobile particles cannot adjust to the excited state of the system after the perturbation, and on the other hand the Hamiltonian of the mobile particles is quadratic for a given disorder configuration. In many situations involving the dynamics of quadratic Hamiltonians, the relaxation results in nonthermal steady states that can be described by a generalized Gibbs ensemble (GGE) [21]. The latter takes into account constraints on the steady state in addition to energy and particle number conservation. A relevant question is therefore whether the trapped state obtained in the DCA can be adequately described by a small number of effective parameters. In an effort to address this question, we study the energy distribution of the trapped states and investigate to what extent the distribution function can be characterized in terms of one or several temperatures and chemical potentials, and whether it is possible to extract a meaningful effective temperature that allows us to explain the values of local and nonlocal observables. 
The rest of this paper is organized as follows. In Sec. II we describe the model and the implementation of the nonequilibrium DCA formalism. In Sec. III we present equilibrium results for different cluster geometries, while Sec. IV is devoted to the nonequilibrium results. Section $\mathrm{V}$ contains a brief conclusion and outlook.

\section{MODEL AND METHOD}

The Falicov-Kimball model [18] was introduced to describe semiconductor-metal transitions in $\mathrm{SmB}_{6}$ and transition-metal oxides. It is similar to the Hubbard model [22] except that it distinguishes localized and itinerant electrons. The Hamiltonian of the (spinless) Falicov-Kimball model with nearest-neighbor hopping and local interactions reads

$$
\mathcal{H}=-t \sum_{\left\langle\mathbf{r}, \mathbf{r}^{\prime}\right\rangle} c_{\mathbf{r}}^{\dagger} c_{\mathbf{r}^{\prime}}+U \sum_{\mathbf{r}}\left(c_{\mathbf{r}}^{\dagger} c_{\mathbf{r}}-\frac{1}{2}\right)\left(f_{\mathbf{r}}^{\dagger} f_{\mathbf{r}}-\frac{1}{2}\right),
$$

where the $c$ electrons are itinerant and the $f$ electrons are localized. Brandt et al. [23-25] derived an exact solution of the Falicov-Kimball model in equilibrium in infinite dimensions using DMFT [26]. Hettler et al. then introduced the DCA $[27,28]$ as an extension to DMFT, which takes nonlocal correlations into account, and they applied it to the FalicovKimball model in two dimensions. Also, the nonequilibrium extension of DMFT was first applied to the Falicov-Kimball model. Freericks and co-workers discussed the damping of Bloch oscillations in the Falicov-Kimball model with static electric fields [1,29], while Eckstein and Kollar [20] studied its relaxation to a nonthermal steady state after an interaction quench. Furthermore, Tsuji et al. [30] studied nonequilibrium steady states in a driven Falicov-Kimball model using Floquet DMFT. In this work, we use a nonequilibrium extension of the DCA formalism for the Falicov-Kimball model to compute the time evolution of local and nonlocal observables after an interaction quench.

In a cluster extension of DMFT [31], one chooses a cluster of lattice sites $\{\tilde{\mathbf{r}}\}$ such that the lattice maps to a superlattice $\{\mathbf{R}\}$ with the clusters as unit cells. In the DCA, we then impose translation invariance under periodic boundary conditions on the cluster, which also leads to a renormalization of the hopping. The reciprocal vectors of the superlattice $\{\tilde{\mathbf{k}}\}$ form patches around the reciprocal vectors of the cluster sites $\{\mathbf{K}\}$; see Fig. 1. Sites on the original lattice are decomposed as
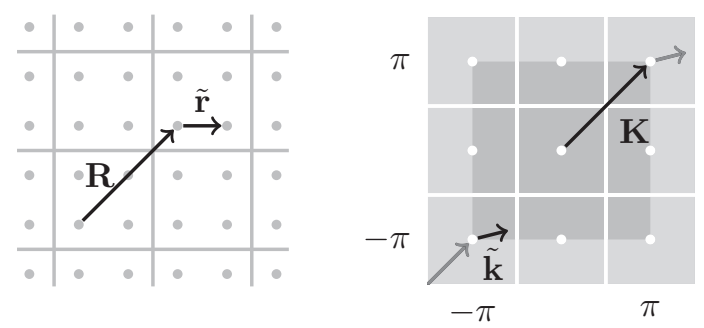

FIG. 1. The sites in a cluster $\tilde{\mathbf{r}}$ form the unit cell of a superlattice $\mathbf{R}$ (left). The first Brillouin zone of the original lattice is split into patches of the size of the first Brillouin zone of the superlattice. The corresponding reciprocal vectors $\tilde{\mathbf{k}}$ are centered around the reciprocal vectors $\mathbf{K}$ of the periodized cluster (right).

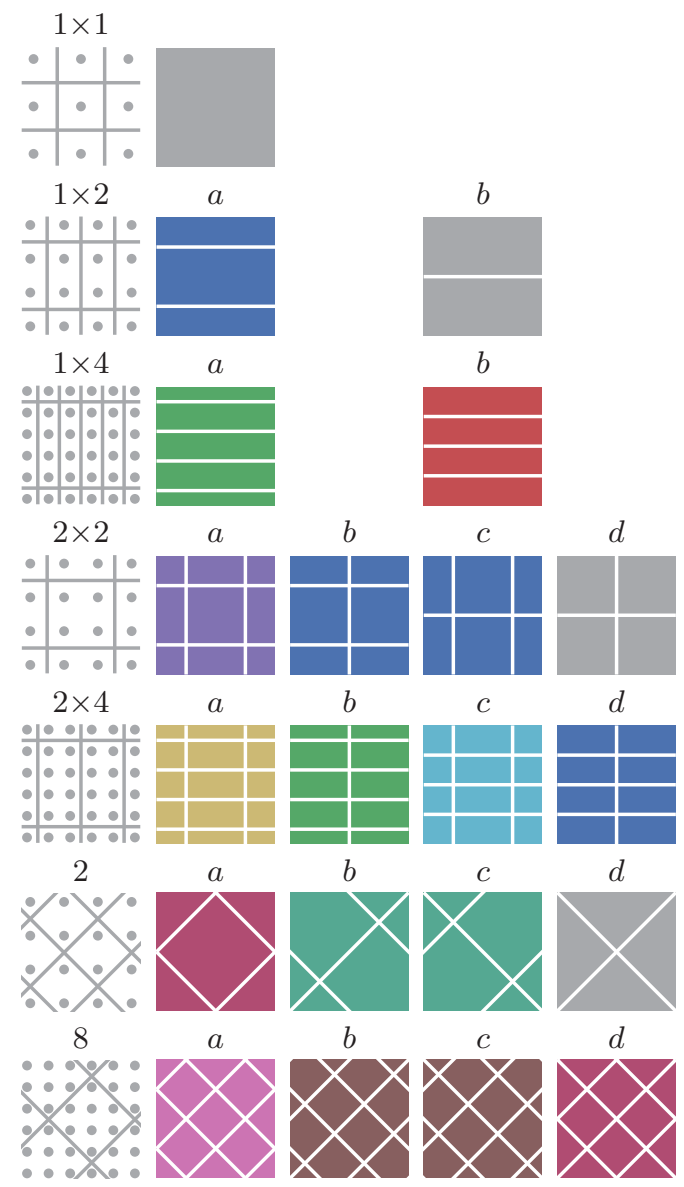

FIG. 2. Cluster geometries and patch layouts considered in this work. The leftmost column depicts different cluster geometries in real space. The remaining columns depict some possible choices of patch layouts in reciprocal space. Patch layouts that are equivalent due to symmetries in the dispersion $\epsilon_{\mathbf{k}}$ are grouped by color.

$\mathbf{r}=\mathbf{R}+\tilde{\mathbf{r}}$, and points in the first Brillouin zone of the original lattice are decomposed as $\mathbf{k}=\mathbf{K}+\tilde{\mathbf{k}}$.

The choice of reciprocal vectors $\{\mathbf{K}\}$ is determined by the cluster shape. However, we are free to choose the layout of the patches that associate the $\mathbf{k}$ vectors in the first Brillouin zone to the reciprocal vectors K. Fig. 2 depicts a number of cluster geometries and corresponding patch layouts in reciprocal space. The leftmost patch layouts represent the canonical choice, where each $\mathbf{k}$ point is associated with the closest $\mathbf{K}$ vector. Some of these patch layouts are equivalent due to symmetries of the dispersion $\epsilon_{\mathbf{k}}$. For example, the layouts $1 \times 1,1 \times 2 b, 2 \times 2 d$, and $2 d$ are equivalent. Simulations on equivalent patch layouts will yield identical results for observables on the whole system, even though the cluster size and number of $f$-particle configurations might differ.

The Falicov-Kimball model (1) maps to the following effective cluster impurity Hamiltonian [31]:

$$
\begin{aligned}
\mathcal{H}_{\mathrm{cl}}-\mu \mathcal{N}_{\mathrm{cl}} & =\mathcal{H}_{0}+\mathcal{H}_{f}+\mathcal{H}_{\text {int }}+\mathcal{H}_{\text {hyb }}+\mathcal{H}_{\text {bath }}, \\
\mathcal{H}_{0} & =\sum_{\mathbf{K}} \bar{\epsilon}_{\mathbf{K}} c_{\mathbf{K}}^{\dagger} c_{\mathbf{K}}-\mu \sum_{\mathbf{K}} c_{\mathbf{K}}^{\dagger} c_{\mathbf{K}},
\end{aligned}
$$




$$
\begin{gathered}
\mathcal{H}_{f}=-\left(\frac{U}{2}+\mu\right) \sum_{\tilde{r}} f_{\tilde{\mathbf{r}}}^{\dagger} f_{\tilde{\mathbf{r}}}, \\
\mathcal{H}_{\text {int }}=\frac{U}{N_{\mathrm{cl}}} \sum_{\mathbf{K}, \mathbf{K}^{\prime}, \tilde{\mathbf{r}}} c_{\mathbf{K}}^{\dagger} c_{\mathbf{K}^{\prime}}\left(f_{\tilde{\mathbf{r}}}^{\dagger} f_{\tilde{\mathbf{r}}}-\frac{1}{2}\right) e^{-i\left(\mathbf{K}-\mathbf{K}^{\prime}\right) \tilde{\mathbf{r}}}, \\
\mathcal{H}_{\text {hyb }}=\sum_{\mathbf{K}, \mathbf{p}}\left(V_{\mathbf{K}, \mathbf{p}} c_{\mathbf{K}}^{\dagger} a_{\mathbf{K}, \mathbf{p}}+\text { H.c. }\right), \\
\mathcal{H}_{\text {bath }}=\sum_{\mathbf{K}, \mathbf{p}} \varepsilon_{\mathbf{K}, \mathbf{p}} a_{\mathbf{K}, \mathbf{p}}^{\dagger} a_{\mathbf{K}, \mathbf{p}},
\end{gathered}
$$

where $\mu$ is the chemical potential, $\mathcal{N}_{\mathrm{cl}}$ is the operator that counts the number of $c$ and $f$ particles, $c^{(\dagger)}$ and $f^{(\dagger)}$ are the (creation) annihilation operators for the mobile and localized electrons on the cluster, $a^{(\dagger)}$ are the bath (creation) annihilation operators, $V_{\mathbf{K}, \mathbf{p}}$ are the hybridization parameters, and $\varepsilon_{\mathbf{K}, \mathbf{p}}$ are the bath energy levels. We also introduce the dispersion of the lattice,

$$
\epsilon_{\mathbf{k}}=-\frac{1}{N} \sum_{\mathbf{r r}^{\prime}} e^{-i \mathbf{k}\left(\mathbf{r}-\mathbf{r}^{\prime}\right)} t_{\mathbf{r r}^{\prime}},
$$

and the patch-averaged dispersion,

$$
\bar{\epsilon}_{\mathbf{K}}=\frac{N_{\mathrm{cl}}}{N} \sum_{\tilde{\mathbf{k}}} \epsilon_{\mathbf{K}+\tilde{\mathbf{k}}},
$$

whose Fourier transform defines the hopping on the periodized cluster.

Furthermore, we introduce the $f$-particle configuration

$$
|\alpha\rangle=\left(\prod_{\tilde{\mathbf{r}}} N_{\alpha \tilde{\mathbf{r}}}^{f} f_{\tilde{\mathbf{r}}}^{\dagger}\right)|0\rangle,
$$

where $N_{\alpha \tilde{\mathbf{r}}}^{f} \in\{0,1\}$ describes the $f$-particle occupation on cluster site $\tilde{\mathbf{r}}$. For a fixed $f$-particle configuration $\alpha$, we can trace out the bath states to obtain the cluster action,

$$
\begin{aligned}
S_{\mathrm{cl}}^{\alpha}= & -\left(\frac{U}{2}+\mu\right) \sum_{\tilde{\mathbf{r}}} N_{\alpha \tilde{\mathbf{r}}}^{f} \\
& -\int_{\mathcal{C}} d z \int_{\mathcal{C}} d z^{\prime} \sum_{\mathbf{K}} \gamma_{\mathbf{K}}^{*}(z) \mathcal{G}_{\mathbf{K}}^{-1}\left(z, z^{\prime}\right) \gamma_{\mathbf{K}}\left(z^{\prime}\right) \\
& +\int_{\mathcal{C}} d z \sum_{\mathbf{K}, \mathbf{K}^{\prime}} \gamma_{\mathbf{K}}^{*}(z) U_{\alpha \mathbf{K} \mathbf{K}^{\prime}} \gamma_{\mathbf{K}^{\prime}}(z),
\end{aligned}
$$

where $\gamma_{\mathbf{K}}$ are the $c$-particle Grassmann numbers,

$$
U_{\alpha \mathbf{K} \mathbf{K}^{\prime}}=\frac{U}{N_{\mathrm{cl}}} \sum_{\tilde{\mathbf{r}}}\left(N_{\alpha \tilde{\mathbf{r}}}^{f}-\frac{1}{2}\right) e^{-i\left(\mathbf{K}-\mathbf{K}^{\prime}\right) \tilde{\mathbf{r}}}
$$

is the interaction matrix for configuration $\alpha$,

$$
\mathcal{G}_{\mathbf{K}}^{-1}\left(z, z^{\prime}\right)=\left(i \partial_{z}-\bar{\epsilon}_{\mathbf{K}}+\mu\right) \delta_{\mathcal{C}}\left(z, z^{\prime}\right)-\Lambda_{\mathbf{K}}\left(z, z^{\prime}\right)
$$

is the inverse excluded-cluster Green's function [31], and $\Lambda_{\mathbf{K}}\left(z, z^{\prime}\right)$ is the hybridization function. The latter two are functions of two variables on the L-shaped contour $\mathcal{C}$, which runs from 0 to $t_{\max }$ and back on the real-time axis, and from 0 to $-i \beta$ on the imaginary-time axis [2].
The cluster Green's function is given by

$$
\begin{gathered}
G_{\mathbf{K}}^{\mathrm{cl}}\left(z, z^{\prime}\right)=\sum_{\alpha} w_{\alpha} R_{\alpha \mathbf{K K}}\left(z, z^{\prime}\right), \\
R_{\alpha \mathbf{K K}^{\prime}}\left(z, z^{\prime}\right)=\frac{\int \mathrm{D}\left[\gamma^{*}, \gamma\right]\left[\mathcal{T}_{\mathcal{C}} e^{-S_{\mathrm{cl}}^{\alpha}} \gamma_{\mathbf{K}}(z) \gamma_{\mathbf{K}^{\prime}}^{*}\left(z^{\prime}\right)\right]}{\int \mathrm{D}\left[\gamma^{*}, \gamma\right]\left[\mathcal{T}_{\mathcal{C}} e^{-S_{\mathrm{cl}}^{\alpha}}\right]}, \\
w_{\alpha}=\frac{\int \mathrm{D}\left[\gamma^{*}, \gamma\right]\left[\mathcal{T}_{\mathcal{C}} e^{\left.-S_{\mathrm{cl}}^{\alpha}\right]}\right]}{\sum_{\alpha} \int \mathrm{D}\left[\gamma^{*}, \gamma\right]\left[\mathcal{T}_{\mathcal{C}} e^{\left.-S_{\mathrm{cl}}^{\alpha}\right]}\right.},
\end{gathered}
$$

where the partial Green's function $R_{\alpha \mathbf{K K}}$ is the $c$-particle Green's function for the fixed $f$-particle configuration $\alpha$, and $w_{\alpha}$ is the weight for this configuration. Evaluating the Gaussian Grassmann integral yields the following contour Fredholm equation for the partial Green's functions in matrix notation:

$$
\left[\mathcal{G}^{-1}-\mathbf{U}_{\alpha}\right]^{-1}=\mathbf{R}_{\alpha}, \quad\left[\mathbb{1}-\mathcal{G} \mathbf{U}_{\alpha}\right] \mathbf{R}_{\alpha}=\mathcal{G} .
$$

Products indicate both matrix multiplication and contour convolution. The cluster Green's function and the excluded-cluster Green's function are diagonal in $\mathbf{K}$, while the interaction matrix and the partial Green's function are not.

In the DCA, we approximate the lattice self-energy by a piecewise-constant function in momentum space, whose values on the different momentum patches (Fig. 2) are given by the cluster self-energy $\Sigma_{\mathbf{K}}=\mathcal{G}_{\mathbf{K}}^{-1}-\left(G_{\mathbf{K}}^{\mathrm{cl}}\right)^{-1}$. We use it to compute an approximate lattice Green's function,

$$
G_{\mathbf{K}+\tilde{\mathbf{k}}}\left(z, z^{\prime}\right)=\left[i \partial_{z}+\mu-\epsilon_{\mathbf{K}+\tilde{\mathbf{k}}}-\Sigma_{\mathbf{K}}\right]^{-1}\left(z, z^{\prime}\right),
$$

and the coarse-grained lattice Green's function,

$$
\bar{G}_{\mathbf{K}}\left(z, z^{\prime}\right)=\frac{N_{\mathrm{cl}}}{N} \sum_{\tilde{\mathbf{k}}} G_{\mathbf{K}+\tilde{\mathbf{k}}}\left(z, z^{\prime}\right) .
$$

The DCA self-consistency condition demands that $\overline{\mathbf{G}}$ is identical to the cluster Green's function $\mathbf{G}^{\mathrm{cl}}$. Thus, we can extract the new cluster-excluded Green's function by solving the Dyson equation

$$
\mathcal{G}^{-1}-\overline{\mathbf{G}}^{-1}=\Sigma, \quad(\mathbb{1}+\overline{\mathbf{G}} \Sigma) \mathcal{G}=\overline{\mathbf{G}} .
$$

Obtaining the cluster self-energy from the cluster Green's function requires the introduction of helper functions (see Appendix A). In practice, we solve the equation

$$
(\mathbb{1}+\mathbf{X G}) \Sigma=\mathbf{X},
$$

where $\mathbf{X G}$ and $\mathbf{X}$ are given by

$$
\begin{aligned}
& \mathbf{X G}=\sum_{\alpha} w_{\alpha} \mathbf{U}_{\alpha} \mathbf{R}_{\alpha}, \\
& \mathbf{X}=\sum_{\alpha} w_{\alpha} \mathbf{U}_{\alpha} \mathbf{R}_{\alpha} \mathbf{U}_{\alpha} .
\end{aligned}
$$

In summary, the DCA solution for the Falicov-Kimball model consists of the following steps: First we obtain a self-consistent solution for the initial equilibrium state. Then we iteratively time-evolve from this equilibrium solution by solving the self-consistency loop at each time step. The steps in the self-consistency loop are as follows:

(i) Start from an initial guess for the self-energy $\Sigma_{\mathbf{K}}\left(z, z^{\prime}\right)$ (usually a zero-order extrapolation from the previous time step). 
(ii) Solve the lattice problem (18) and compute the coarsegrained lattice Green's function $\bar{G}_{\mathbf{K}}\left(z, z^{\prime}\right)$.

(iii) Obtain $\mathcal{G}_{\mathbf{K}}\left(z, z^{\prime}\right)$ from Eq. (20).

(iv) Solve the cluster problem and calculate $G_{\mathbf{K}}^{\mathrm{cl}}\left(z, z^{\prime}\right)$ from Eqs. (14) and (17). (The configuration weights $w_{\alpha}$ only have to be calculated in the initial equilibrium state, since they are time-independent.)

(v) Obtain the self-energy $\Sigma_{\mathbf{K}}\left(z, z^{\prime}\right)$ from Eq. (21).

(vi) Start over with step (ii) until convergence. Then start over with step (i) at the next time step.

\section{EQUILIBRIUM}

\section{A. Spectral function}

To determine the spectral function (Fig. 3), we time-evolve the equilibrium system up to $t_{\max }=30$, Fourier transform the retarded component of the Green's function

$$
G^{R}(\omega)=\int_{t_{\mathrm{start}}}^{t_{\max }} d t G^{R}\left(t, t_{\mathrm{start}}\right) e^{i \omega\left(t-t_{\mathrm{start}}\right)},
$$
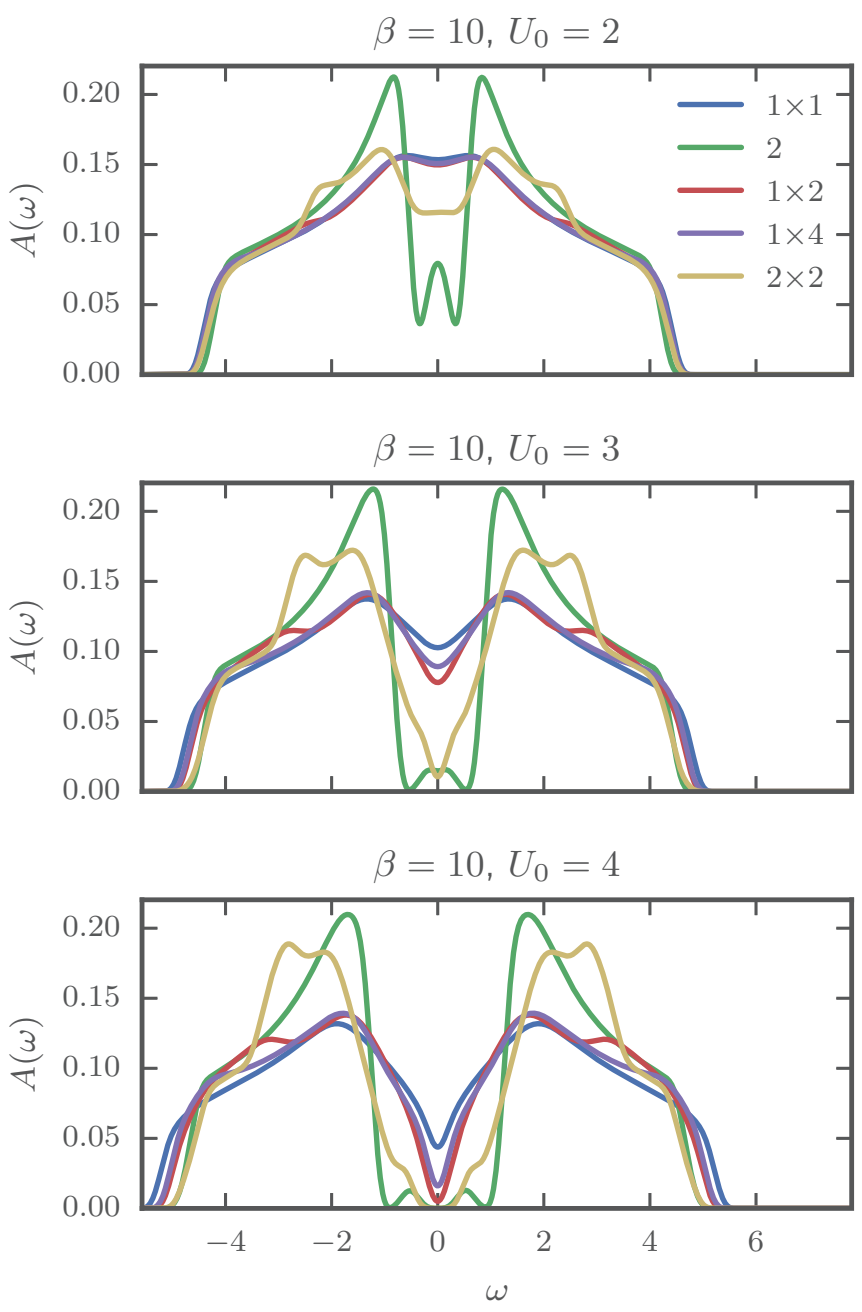

FIG. 3. Comparison of the spectral function for different cluster geometries and interaction parameters in equilibrium. In all cases, the canonical patch layout was used. where $t_{\text {start }}=0$, and use that

$$
A(\omega)=-\frac{1}{\pi} \operatorname{Im} G^{R}(\omega) .
$$

We observe the opening of a gap in the range $3 \lesssim U \lesssim 4$. The insulating nature is stronger when nonlocal correlations are included, which may be attributed to a charge-ordering tendency (see the next paragraph). However, the " 2 " cluster, and to a lesser extent the $2 \times 2$ cluster, overestimate these charge-order correlations, and hence the gap. Additional $K$ patches add features to the spectral function, some of which are artefacts of the piecewise-constant self-energy. Larger clusters than shown in Fig. 3 would be needed for a converged solution. (Results for the eight-site cluster are not shown, because memory restrictions do not allow us to time-propagate to $t=30$. Hence, we cannot reach the same spectral resolution as for the smaller clusters.)

\section{B. Local and nonlocal observables}

The cluster Green's function contains nonlocal components and hence gives access to nonlocal observables. For example, we can calculate the nearest-neighbor density-density correlations between $c$ and $f$ particles on the cluster as follows:

$$
\left\langle n_{\tilde{\mathbf{r}}}^{c} n_{\tilde{\mathbf{r}}^{\prime}}^{f}\right\rangle=\sum_{\alpha} w_{\alpha}\left\langle c_{\tilde{\mathbf{r}}}^{\dagger} c_{\tilde{\mathbf{r}}}\right\rangle_{\alpha} N_{\alpha \tilde{\mathbf{r}}^{\prime}}^{f}=\sum_{\alpha} w_{\alpha} \operatorname{Im} R_{\alpha \tilde{\mathbf{r}} \tilde{\mathbf{r}}}^{<} N_{\alpha \tilde{\mathbf{r}}^{\prime}}^{f}
$$

Some cluster layouts break the symmetry between nearestneighbor pairs along the horizontal or vertical axis. To mitigate this effect, it is useful to average over all nearest-neighbor pairs in the cluster, including those due to periodic boundary conditions. If there is no nearest neighbor along a given axis, as, for example, along the horizontal axis in the $1 \times 2$ cluster, then we apply the mean-field approximation $\left\langle n_{\tilde{\mathbf{r}}^{\prime}}^{c} n_{\tilde{\mathbf{r}}^{\prime}}^{f}\right\rangle \approx$ $\left\langle n_{\tilde{\mathbf{r}}}^{c}\right\rangle\left\langle n_{\tilde{\mathbf{r}}^{\prime}}^{f}\right\rangle$. In this way, we obtain nearest-neighbor densitydensity correlations between $c$ and $f$ particles.

Nearest-neighbor density-density correlations between $c$ particles are obtained by applying Wick's theorem to the expectation value for each fixed $f$-particle configuration:

$$
\begin{aligned}
\left\langle n_{\tilde{\mathbf{r}}}^{c} n_{\tilde{\mathbf{r}}^{\prime}}^{c}\right\rangle= & \sum_{\alpha} w_{\alpha}\left\langle c_{\tilde{\mathbf{r}}}^{\dagger} c_{\tilde{\mathbf{r}}} c_{\tilde{\mathbf{r}}^{\prime}}^{\dagger} c_{\tilde{\mathbf{r}}^{\prime}}\right\rangle_{\alpha} \\
= & \sum_{\alpha} w_{\alpha}\left[\left\langle c_{\tilde{\mathbf{r}}}^{\dagger} c_{\tilde{\mathbf{r}}}\right\rangle_{\alpha}\left\langle c_{\tilde{\mathbf{r}}^{\prime}}^{\dagger} c_{\tilde{\mathbf{r}}^{\prime}}\right\rangle_{\alpha}+\left\langle c_{\tilde{\mathbf{r}}}^{\dagger} c_{\tilde{\mathbf{r}}^{\prime}}\right\rangle_{\alpha}\left\langle c_{\tilde{\mathbf{r}}} c_{\tilde{\mathbf{r}}^{\prime}}^{\dagger}\right\rangle_{\alpha}\right] \\
= & \sum_{\alpha} w_{\alpha}\left[\operatorname{Im} R_{\alpha}^{<} R_{\tilde{\mathbf{r}} \tilde{\mathbf{r}}} \operatorname{Im} R_{\alpha \tilde{\mathbf{r}}^{\prime} \tilde{\mathbf{r}}^{\prime}}^{<}\right. \\
& \left.+\operatorname{Im} R_{\alpha}^{<} \tilde{\mathbf{r}}^{\prime} \tilde{\mathbf{r}}^{\prime}\left(\delta_{\tilde{\mathbf{r}} \tilde{\mathbf{r}}^{\prime}}-\operatorname{Im} R_{\alpha}^{<} \tilde{\mathbf{r}}^{\prime}\right)\right] .
\end{aligned}
$$

We apply the same averaging over nearest-neighbor pairs as described in the previous paragraph, including the mean-field approximation, if there are no nearest neighbors along a certain axis.

Nearest-neighbor density-density correlations between $f$ particles are obtained in the same way. However, since there are no off-diagonal contributions to the occupation operator, the expectation value simplifies to the following expression:

$$
\left\langle n_{\tilde{\mathbf{r}}}^{f} n_{\tilde{\mathbf{r}}^{\prime}}^{f}\right\rangle=\sum_{\alpha} w_{\alpha} N_{\alpha \tilde{\mathbf{r}}}^{f} N_{\alpha \tilde{\mathbf{r}}^{\prime}}^{f} .
$$



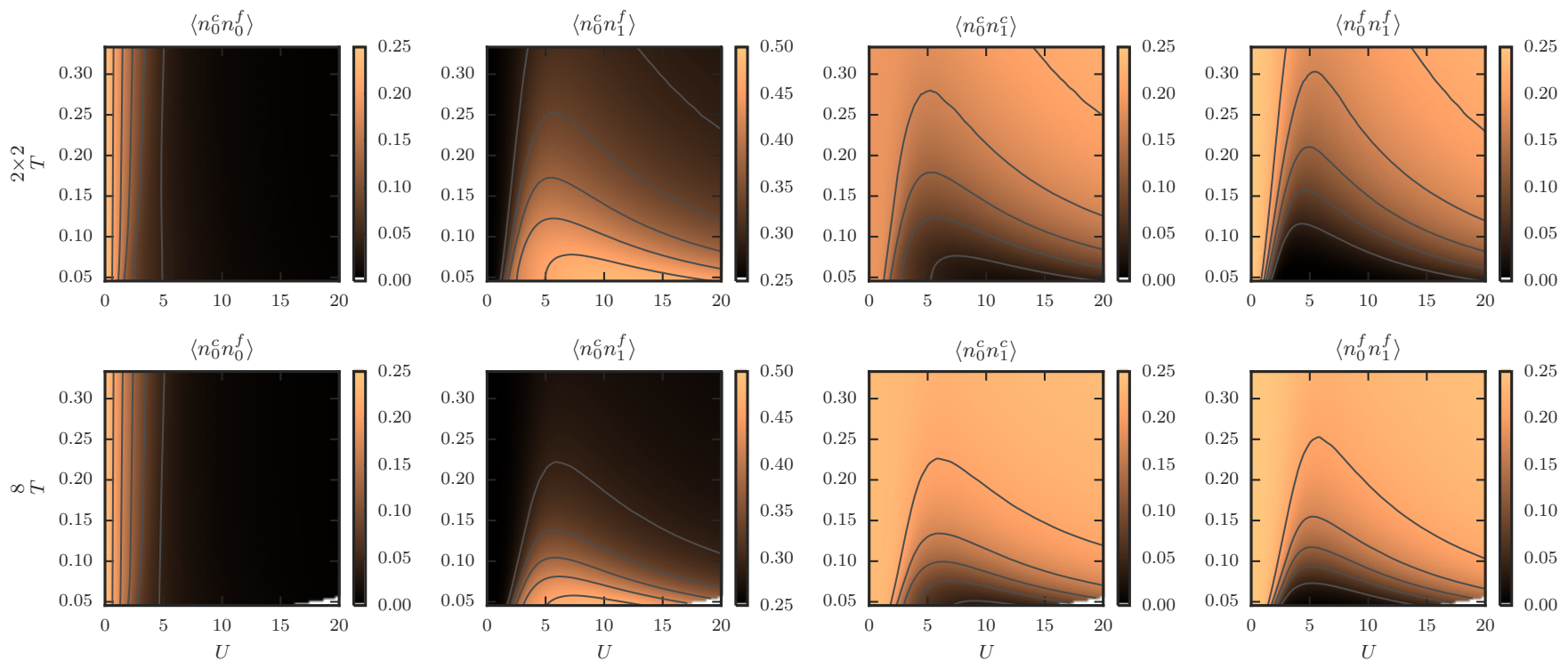

FIG. 4. Local and nonlocal cluster correlations in equilibrium at different temperatures and interaction strengths. The first column depicts the double occupation, and the next three columns depict nearest-neighbor density-density correlations between $c$ - and $f$-, $c$ - and $c$-, and $f$ and $f$-particles, respectively. The upper row corresponds to the $2 \times 2$ cluster, and the lower row corresponds to the eight-site cluster. In both cases, the canonical patch layout was used.

It should be emphasized that due to the periodization in the DCA, the nearest-neighbor cluster correlation functions are not identical to the corresponding lattice quantities. However, for large enough clusters, the cluster correlations should provide a good estimate, so that it is meaningful to study the convergence with cluster size.

Local observables such as the double occupation can be obtained in the same manner:

$$
\left\langle n_{\tilde{\mathbf{r}}}^{c} n_{\tilde{\mathbf{r}}}^{f}\right\rangle=\sum_{\alpha} w_{\alpha} \operatorname{Im} R_{\alpha \tilde{\mathbf{r}} \mathbf{r}}^{<} N_{\alpha \tilde{\mathbf{r}}}^{f}
$$

In the case of local quantities, the DCA self-consistency condition guarantees that the cluster observables coincide with the lattice observables.

Equilibrium results of these correlation functions are depicted in Fig. 4 for the $2 \times 2$ and the eight-site cluster. The double occupation indicates a metal-insulator transition with weak temperature dependence, while the nonlocal correlations show a tendency toward charge order at low temperature, which is overestimated in the $2 \times 2$ case. We do not explicitly break translation invariance. Therefore, we cannot observe an actual charge-order phase transition. Nevertheless, our results are compatible with the results by Hettler et al. $[19,28]$ (for the symmetry-broken phase) except for the $(2 \times 2)$-site cluster, which shows the strongest suppression of charge order in their study. Our correlation functions show the opposite effect, namely an enhancement of the charge-order correlations in the canonical $2 \times 2$ geometry, which also explains the overestimation of the gap in Fig. 3.

A more systematic trend with cluster size can be obtained by averaging over the different patch layouts shown in Fig. 2 . After this averaging, the charge-order correlations of the $2 \times 2$ cluster become weaker than in the eight-site cluster.

\section{NONEQUILIBRIUM}

\section{A. Interaction ramp}

To investigate the nonequilibrium dynamics of the system, we start in an equilibrium state at finite temperature and change the interaction parameter according to the protocol

$$
U(t)=U_{0}+\left(U_{q}-U_{0}\right) r\left(\frac{t}{t_{\text {ramp }}}-t_{0}\right),
$$

with the ramp shape function

$$
r(x)= \begin{cases}0, & x<0, \\ \frac{1}{2}-\frac{3}{4} \cos (\pi x)+\frac{1}{4} \cos ^{3}(\pi x), & 0 \leqslant x<1, \\ 1, & 1 \leqslant x .\end{cases}
$$

The ramp begins at $t_{0}$ and switches the interaction parameter from its initial value $U_{0}$ to the final value $U_{q}$ in a time $t_{\text {ramp }}$. The smooth shape of the ramp function helps to reduce the energy injected into the system. Throughout this section, we choose $t_{0}=0$ and $t_{\text {ramp }}=3$.

The time evolution of local and nonlocal correlation functions is shown in Fig. 5 for a ramp from $U_{0}=3$ to $U_{q}=4$. Here, we averaged the results over the different patch layouts depicted in Fig. 2. In the case of a one-dimensional (1D) Hubbard system, this type of averaging was found to improve the accuracy of the time evolution [12]. Also for the present model and ramp setup, it turns out that the averaging over patch layouts results in a much more systematic trend with cluster size, even though an actual convergence cannot yet be observed with clusters up to eight sites.

The increase of $U$ moves the system further into the insulating regime, as confirmed by all three correlation functions, and in particular by a reduction of the double occupancy. The larger clusters exhibit both stronger initial nonlocal correlations and a stronger buildup of additional 

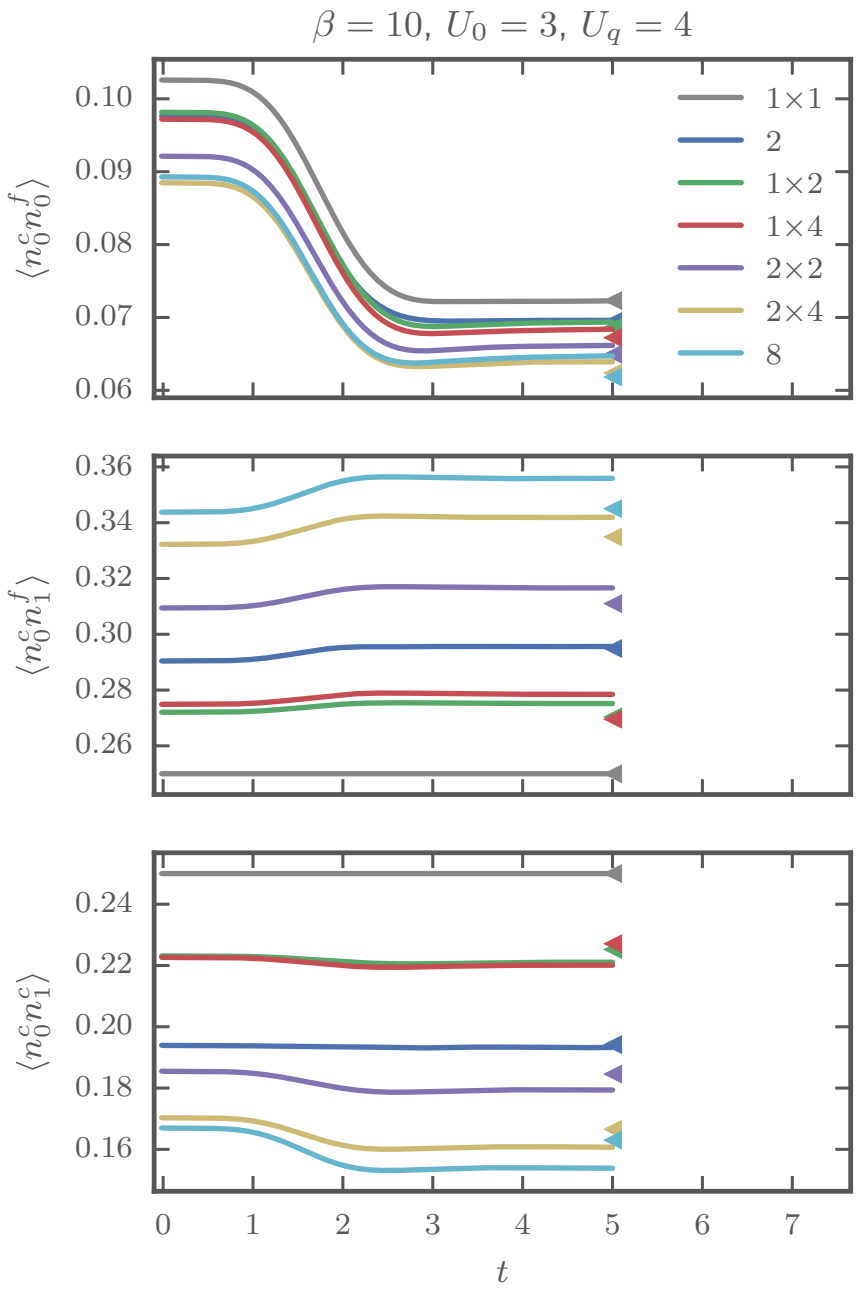

FIG. 5. Local and nonlocal correlations between $c$ and $f$ particles after an interaction ramp from $U_{0}=3$ to $U_{q}=4$, starting from an equilibrium state at $\beta=10$. Results are shown for different cluster geometries, averaged over different patch layouts. The uppermost plot depicts the local double occupation. The middle plot depicts nearestneighbor density-density correlations between $c$ and $f$ particles. The lowermost plot depicts nearest-neighbor density-density correlations between $c$ particles. The triangles to the right indicate the expectation values in an equilibrium system with the same total energy.

nonlocal correlations during the ramp. After the ramp, the system relaxes to a nonthermal steady state. The small triangles depict the expectation values for an equilibrium system with the same total energy. If the nonequilibrium system were to thermalize, then the observables would converge to these results. Evidently, the DCA simulations do not thermalize, as expected for the Falikov-Kimball model, in which the distribution of $f$ particles cannot react to the change in energy.

While the reduction of the double occupancy during the ramp is at least roughly consistent with the expected changes in a thermalizing system, the enhanced correlations in the nonlocal observables reflect a deviation from thermal equilibrium (apart from the $1 \times 1$ cluster, where nonlocal correlations trivially factorize, $\left\langle n_{0}^{c} n_{1}^{f}\right\rangle=\left\langle n^{c}\right\rangle\left\langle n^{f}\right\rangle$ ). To explain this effect and to systematically investigate the deviation between the trapped state in the long-time limit and the
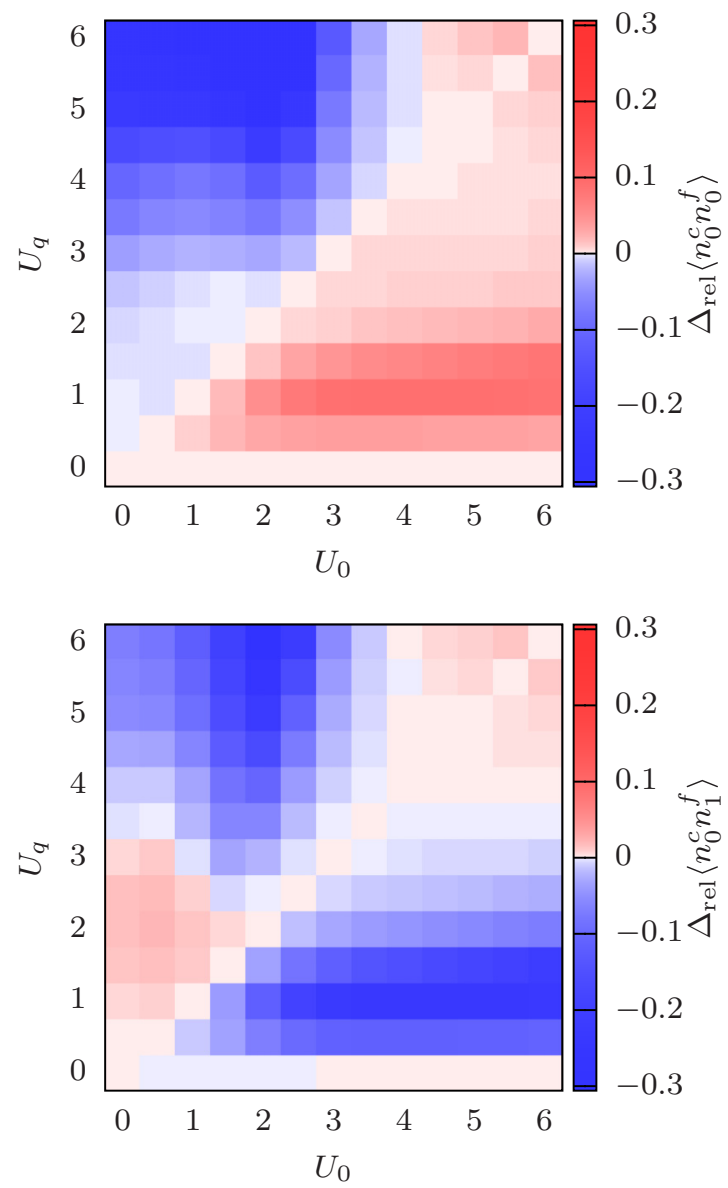

FIG. 6. Difference between the thermal expectation values and the steady-state observables [Eq. (32)] after an interaction ramp $(2 \times 2$ cluster, canonical patch layout, initial $\beta=10)$. The system is ramped from $U_{0}$ to $U_{q}$ according to Eq. (30), and the double occupancy and $c$ - $f$ nearest-neighbor density-density correlation are measured. The color scale in the double-occupancy plot is cropped to match the scale of the nearest-neighbor correlation plot.

corresponding equilibrium state with identical energy, we have run a series of calculations for different initial interactions $U_{0}$ and final interactions $U_{q}$ for the $2 \times 2$ cluster at initial inverse temperature $\beta=10$. As a local observable, we consider the double occupation $\left\langle n_{0}^{c} n_{0}^{f}\right\rangle$ and as a nonlocal observable the $c-f$ nearest-neighbor correlation $\left\langle n_{0}^{c} n_{1}^{f}\right\rangle$. The expectation values of the trapped state are measured at $t=20$, and the relative difference to the thermal values is computed as

$$
\Delta_{\text {rel }} O \equiv \frac{O_{\text {th }}-O}{O_{\text {th }}} .
$$

Figure 6 shows the measured deviations as intensity plots in the space of $U_{0}$ and $U_{q}$. Remembering that the critical interaction for the metal-insulator transition in the $2 \times 2$ cluster is about $U_{c} \approx 3$ (Fig. 3), we notice that for ramps within the metallic regime $\left(U_{0}, U_{q}<U_{c}\right)$ or within the insulating regime $\left(U_{0}, U_{q}>U_{c}\right)$, the double occupation reaches a value close to the thermal one, while the thermal value substantially overestimates the trapped double occupancy after up-ramps across $U_{c}$. Similarly, the thermal value substantially underestimates the double occupancy after down-ramps across $U_{c}$, except 
near $U_{q}=0$, where the correct double occupation of 0.25 is reproduced.

The result for $\left\langle n_{0}^{c} n_{1}^{f}\right\rangle$ is similar to that for the double occupation, except that the sign of the relative change is opposite for the case of ramps into the metal regime. For quenches across $U_{c}$ (independent of the quench direction), the short-range charge order is stronger in the nonthermal state than it would be after thermalization. This can be understood, because a higher temperature reduces the shortrange correlations. Even in a coupling regime where a change of the interaction from $U_{0}$ to $U_{q}$ at fixed temperature would increase the charge correlations, the energy injected into the system can (if thermalized) more than compensate for this and results in weaker correlations. One may furthermore wonder why nearest-neighbor charge correlations can increase at all after the quench (as in Fig. 5), although the $f$ particles are static. This occurs because already in the initial state there is a short-range charge order, which implies nearest-neighbor anticorrelations between the $f$ particles. Since the $f$ particles are frozen, this short-range order remains after the quench. The nearest-neighbor $c$ - $f$ correlations are hence likely to increase if an increase of $U$ leads to a reduction in the double occupancy, i.e., an increase in the density of $c$ particles at sites that are not occupied by $f$ particles.

The sign change in $\Delta_{\text {rel }}\left\langle n_{0}^{c} n_{0}^{f}\right\rangle$ near $U_{q}=U_{c}\left(U_{c} \approx 3\right.$ is the critical value of the Mott transition) results in small deviations between thermal and trapped nearest-neighbor $c-f$ correlations after quenches to $U_{q} \approx U_{c}$. As we will see in the following section, this does not mean, however, that the energy distribution functions after such quenches are close to thermal distributions.

\section{B. Effective temperatures}

In the absence of thermalization, an interesting issue is whether or not the state of the system can be characterized by a small number of parameters, such as effective temperatures or effective chemical potentials. In fact, since the FalicovKimball lattice model has a large number of conserved quantities, an exact description in terms of a generalized Gibbs ensembles (GGE) is possible (see Appendix B). However, a GGE description with an extensive number of parameters is not very useful, and it is also not clear how this construction can be adapted to the DCA case.

In DCA, the $f$-particle configurations are conserved by the time evolution, and one possible goal could be to devise a GGE-like description of the trapped state that is based on effective temperatures and chemical potentials that depend on the $f$ configuration. With this motivation in mind, we will investigate in the following sections to what extent the notion of an effective ( $c$-electron) temperature is useful to characterize the trapped states observed in DCA simulations.

To address this issue, we consider the quantity

$$
h(\omega)=\log \left[-2 \operatorname{Im} G^{R}(\omega) / \operatorname{Im} G^{<}(\omega)-1\right],
$$

which turns out to be independent of the real-space components or cluster momenta. In thermal equilibrium, one has $G^{<}(\omega)=2 \pi i A(\omega) f(\omega)$ due to the fluctuation-dissipation theorem, so that $h(\omega)$ will linearly increase with a slope given by $\beta=1 / T$. In the nonequilibrium case, the slope of $h(\omega)$ yields a possible definition of an effective inverse temperature $\beta_{\text {eff }} \equiv 1 / T_{\text {eff. }}$. In Eq. (33), we do not show a time argument because we assume that the spectral functions are computed in the nonthermal steady state reached after the quench. (For the results shown in Fig. 7, we have propagated the solution up to $t=80$ and computed the spectral functions by Fourier transformation over the time interval $[40,80]$.)

In the following, we focus on the $2 \times 2$ cluster and choose a relatively high initial temperature $\beta=2$. Figure 7 plots the spectral function, the occupation function, and the quantity (33) for quenches from $U_{0}=2$ to $U_{q}=3, U_{0}=3$ to $U_{q}=2$, $U_{0}=2$ to $U_{q}=5$, and $U_{0}=5$ to $U_{q}=2$. The two panels correspond to the $k=(\pi, \pi)$ and $k=(0, \pi)$ components. Error bars on $h$ were estimated by error propagation from the error $\sigma_{A}$ on the spectral functions, which is mainly due to the finite time interval of the Fourier transform. (The spectral function should integrate to 1 , and we used the deviation in that integral to estimate $\sigma_{A}$.) From the slope of $h(\omega)$, we extract the "energy-dependent effective temperature" $[d h(\omega) / d \omega]^{-1}=T_{\text {eff }}(\omega)$, which is overlaid on the spectra in the left panels (green curves, right scale). Within the accuracy of our calculation, the effective temperatures are the same for all $k$ patches. After the quench from $U=3 \rightarrow 2$, $h(\omega)$ exhibits an approximately linear $\omega$ dependence, roughly consistent with a thermal distribution, although there are flat regions near $\omega= \pm 1.5$ (resulting in a noticeable increase of the inverse slope $\left.[d h(\omega) / d \omega]^{-1}\right)$. After the $U=5 \rightarrow 2$ ramp, we observe pronounced spikes in $[d h(\omega) / d \omega]^{-1}$ at the same energies. Similarly, after the quenches from $U=2$ to larger interactions, the effective temperature profile shows large variations as a function of $\omega$. In particular, we note that even after the $U=2 \rightarrow 3$ ramp, for which the local and nonlocal observables shown in Fig. 6 are close to their thermal counterparts, $T_{\text {eff }}(\omega)$ exhibits a large $\omega$ dependence, and therefore the steady state cannot be described by a single effective temperature.

In general, one observes that the $h(\omega)$ curves feature offsets between different almost-linear intervals. In each frequency interval with a linear slope of $h(\omega)$, the distribution function could be parametrized by a Fermi function with some effective chemical potential. Connecting two regions with different chemical potential offsets gives rise to plateaus in $h(\omega)$, which in turn cause spikes in the "temperature profile."

By taking the mode (most common value) of $\beta_{\text {eff }}(\omega)$ over the energy range in which this quantity can be accurately determined, we obtain $\bar{\beta}_{\text {eff }} \equiv 1 / \bar{T}_{\text {eff }}$, which may be regarded as a possible definition of the effective temperature of the trapped state. The modal value is not sensitive to the pronounced spikes in $[d h(\omega) / d \omega]^{-1}$. In Table I, we compare the double occupancies and $\left\langle n_{0}^{c} n_{1}^{f}\right\rangle$ expectation values at $\bar{\beta}_{\text {eff }}$ to the trapped values, and to the thermal values that the system would reach if it could thermalize at the given energy. It is evident that $\bar{\beta}_{\text {eff }}$ does not provide a particularly accurate description of the observables in the trapped state; the predictions based on $\bar{\beta}_{\text {eff }}$ are generally worse than those based on the effective thermal temperature $T_{\mathrm{th}}=1 / \beta_{\mathrm{th}}$. This poor result is probably related to the fact that our effective thermal description ignores the fact that $h(\omega)$ is only piecewise (approximately) linear, i.e., different energy intervals have different effective chemical potentials. 

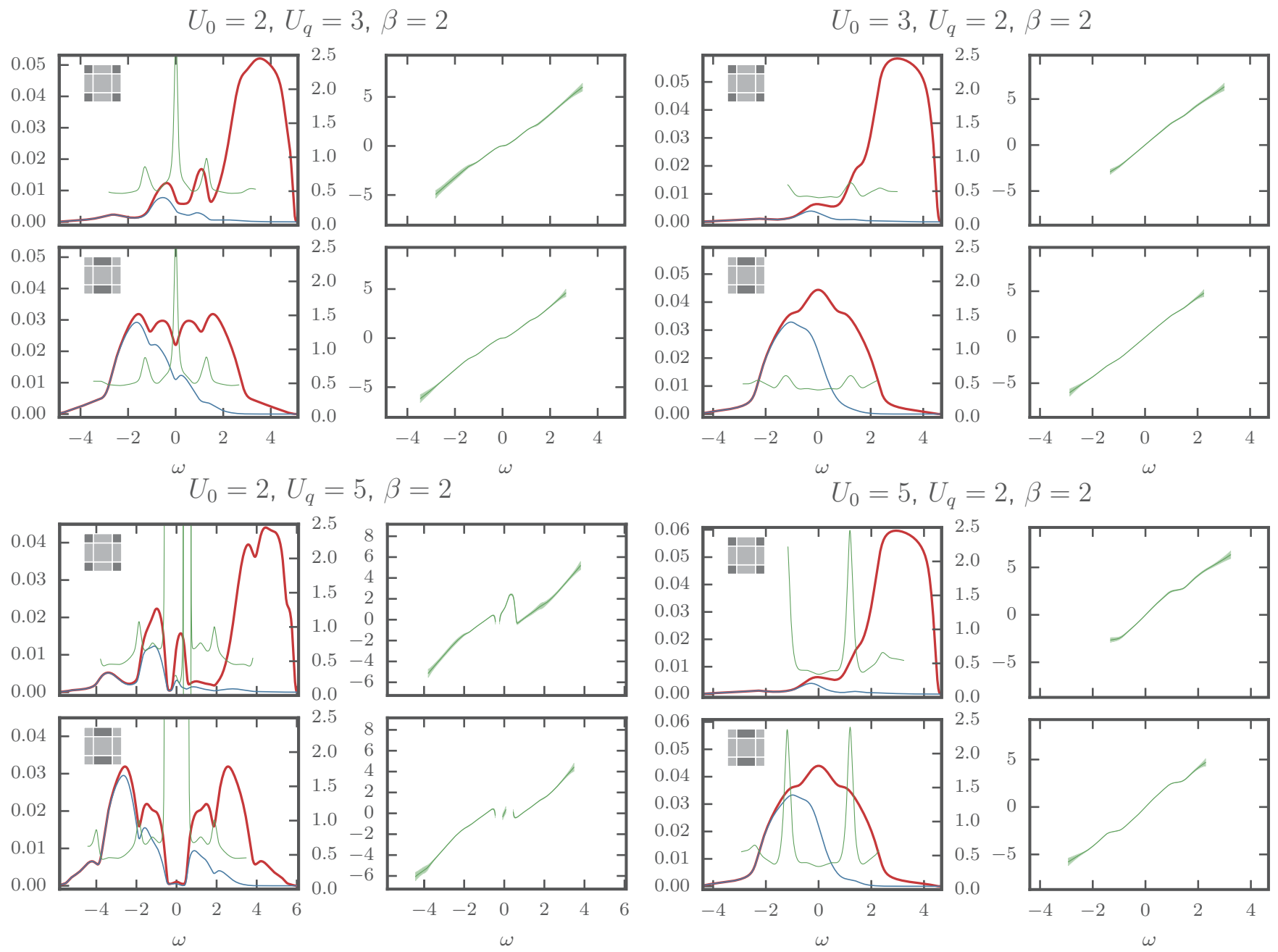

FIG. 7. Spectral and occupation functions for different $K$ patches after ramping the interaction parameter from $U_{0}$ to $U_{q}$ within the time interval $[0,3]$. The thick red line is the spectral function, and the thin blue line is the occupation function. The green line in the right panels depicts the function $h(\omega)$ whose slope would correspond to the inverse temperature $\beta$ in a thermalized system. The thin green line that is laid over the spectral function shows the reciprocal of that slope.

To get more insights into the $\omega$ dependence of $T_{\text {eff }}$, we consider the ramp from $U=5 \rightarrow 2$, which yields large spikes at the energies $\omega \approx \pm 1.2$ (Fig. 7), and we calculate the contributions to the spectral function from the different $f$-particle configurations. Figure 8 shows the results for the following configurations (from top to bottom): No $f$ particles, one $f$ particle, two particles along an edge, two particles along a diagonal, three $f$ particles, fully occupied.
We observe that the frequency-dependent effective temperature is identical for all configurations, and hence identical to the (momentum-independent) effective temperature in Fig. 7. One can indeed show rigorously that if the distribution function of the cluster Green's function is independent of momentum, then also the Green's function $\mathbf{R}_{\alpha}$ for each individual $f$ particle configuration must have the same distribution function. Let us assume that the system has relaxed to a steady state

TABLE I. Long-time expectation values and inverse temperatures corresponding to Fig. 7. The column $\beta$ indicates the inverse temperature before the ramp, $\beta_{\mathrm{th}}$ is the inverse temperature at which an equilibrium system with otherwise identical parameters has the same total energy as the system after the ramp, while $\bar{\beta}_{\text {eff }} \equiv 1 / \bar{T}_{\text {eff }}$ is obtained from the mode (most common value) of the inverse temperatures extracted from the slope of $h(\omega)$.

\begin{tabular}{|c|c|c|c|c|c|c|c|c|c|c|}
\hline Cluster & $U$ & $\beta$ & $\left\langle n_{0}^{c} n_{0}^{f}\right\rangle$ & $\left\langle n_{0}^{c} n_{1}^{f}\right\rangle$ & $\beta_{\text {th }}$ & $\left\langle n_{0}^{c} n_{0}^{f}\right\rangle_{\text {th }}$ & $\left\langle n_{0}^{c} n_{1}^{f}\right\rangle_{\mathrm{th}}$ & $\bar{\beta}_{\text {eff }}$ & $\left\langle n_{0}^{c} n_{0}^{f}\right\rangle_{\mathrm{eff}}$ & $\left\langle n_{0}^{c} n_{1}^{f}\right\rangle_{\text {eff }}$ \\
\hline $2 \times 2$ & $2 \rightarrow 3$ & 2 & 0.121 & 0.265 & 1.56 & 0.12 & 0.266 & 1.72 & 0.117 & 0.268 \\
\hline $2 \times 2$ & $2 \rightarrow 5$ & 2 & 0.0992 & 0.273 & 0.905 & 0.0856 & 0.261 & 1.45 & 0.0633 & 0.273 \\
\hline $2 \times 2$ & $3 \rightarrow 2$ & 2 & 0.151 & 0.268 & 2.3 & 0.152 & 0.264 & 2.09 & 0.154 & 0.263 \\
\hline $2 \times 2$ & $5 \rightarrow 2$ & 2 & 0.149 & 0.277 & 2.36 & 0.152 & 0.264 & 2.02 & 0.154 & 0.263 \\
\hline
\end{tabular}



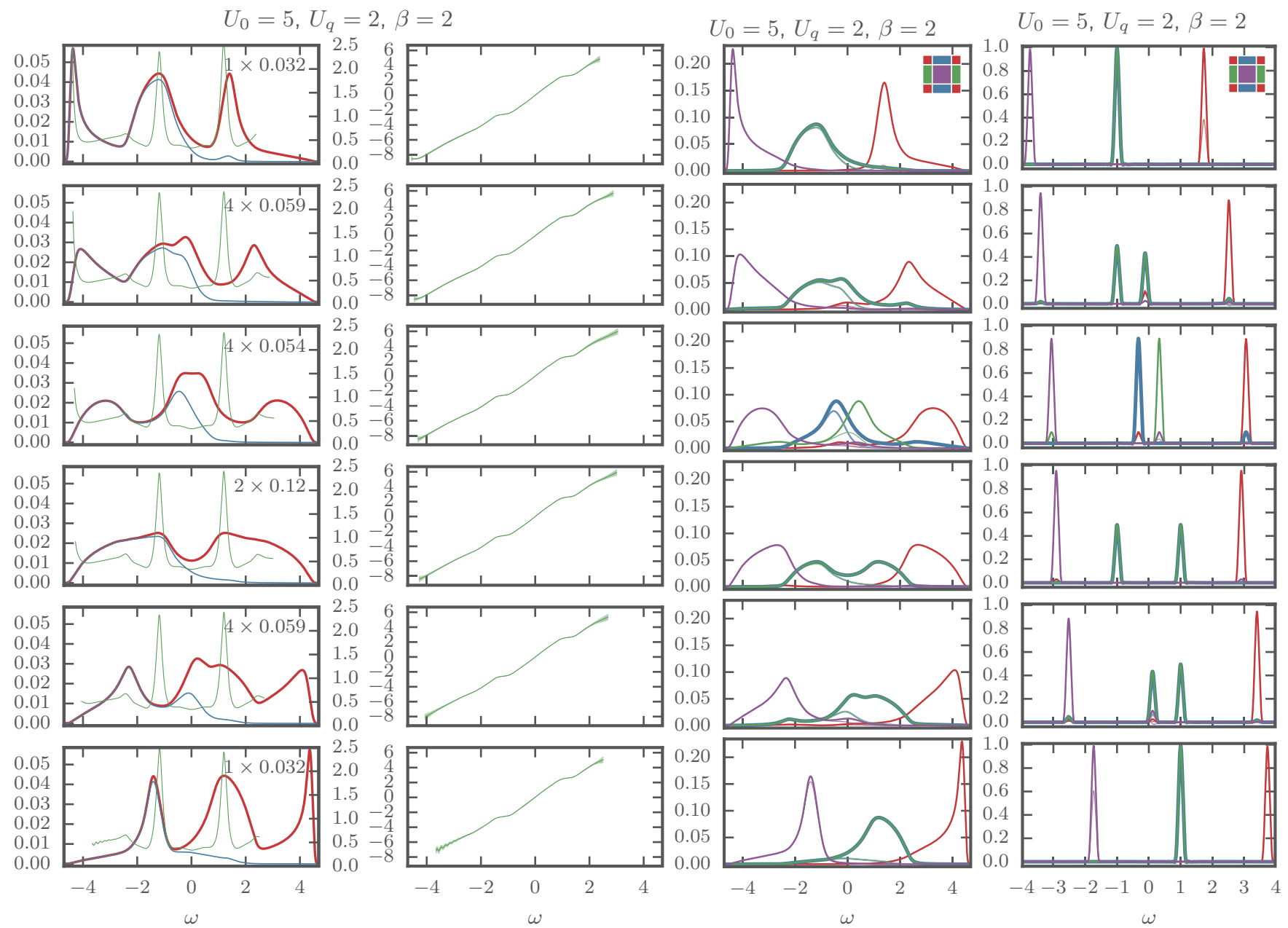

FIG. 8. First column: Spectral and occupation functions for different $f$-particle configurations after ramping the interaction parameter from $U_{0}$ to $U_{q}$ within the time interval [0,3]. The thick red line is the spectral function, and the thin blue line is the occupation function. Second column: Function $h(\omega)$, whose slope would correspond to the inverse temperature $\beta$ in a thermalized system. The thin green line that is laid over the spectral function in the left panels shows the reciprocal of that slope, i.e., $T_{\text {eff }}(\omega)$ (right axis). The following $f$-particle configurations are depicted from top to bottom: Unoccupied, one $f$ particle, two particles along an edge, two particles along a diagonal, three $f$ particles, fully occupied. The numbers in the upper right corner of each panel in the first column denote the multiplicity of that configuration due to symmetry and the corresponding configuration weight $w_{\alpha}$. Third column: Spectral and occupation functions for different $f$-particle configurations and momentum patches. The spectra in the first column are the normalized sum of these. Fourth column: Energy spectrum for different $f$-particle configurations and momentum patches in an isolated cluster at $U=2$. The $(0, \pi)$, and $(\pi, 0)$ spectra (green and blue curves) overlap in all but the third row.

in which the contour objects $\mathcal{G}$ and $\mathbf{R}_{\alpha}$ can be characterized by $\omega$-dependent retarded, advanced, and Keldysh components. We can then use the parametrization

$$
\begin{aligned}
\mathcal{G}^{K} & =\mathcal{G}^{R} \mathbf{F}_{\mathcal{G}}+\mathbf{F}_{\mathcal{G}} \mathcal{G}^{A}, \\
\mathbf{R}_{\alpha}^{K} & =\mathbf{R}_{\alpha}^{R} \mathbf{F}_{\alpha}+\mathbf{F}_{\alpha} \mathbf{R}_{\alpha}^{A},
\end{aligned}
$$

where $\mathbf{F}$ is the nonthermal distribution function. Inserting the ansatz (34) into Eq. (17), we obtain the Keldysh component of the partial Green's function,

$$
\begin{aligned}
\mathbf{R}_{\alpha}^{K} & =\mathbf{R}_{\alpha}^{R} \mathcal{G}^{R^{-1}} \mathcal{G}^{K} \mathcal{G}^{A^{-1}} \mathbf{R}_{\alpha}^{A} \\
& =\mathbf{R}_{\alpha}^{R} \mathbf{F}_{\mathcal{G}}-\mathbf{F}_{\mathcal{G}} \mathbf{R}_{\alpha}^{A}+\mathbf{R}_{\alpha}^{R}\left[\mathbf{F}_{\mathcal{G}}, \mathbf{U}_{\alpha}\right] \mathbf{R}_{\alpha}^{A},
\end{aligned}
$$

where $[\cdot, \cdot]$ is the commutator. If the distribution function $\mathbf{F}_{\mathcal{G}}$ is momentum-independent, then $\mathbf{F}_{\mathcal{G}}$ is proportional to the identity matrix and commutes with the interaction matrix $\mathbf{U}_{\alpha}$. It then follows from a comparison to Eq. (35) that $\mathbf{F}_{\alpha}=\mathbf{F}_{\mathcal{G}}$, i.e., the distribution is also configuration-independent.

The spectral functions for a fixed $f$-particle configuration consist of subbands that can be identified with certain $K$ patches, as depicted in the third column of Fig. 8. We observe that the plateaus in $h(\omega)$, between energy regions with a different chemical potential, occur at the boundaries between these subbands. In particular, we can associate them with the region between the $(\pi, \pi)$ subband and the subbands corresponding to $K=(0, \pi),(\pi, 0)$ in the configurations with one $f$ particle (second row), and the region between the $(0,0)$ subband and the $K=(0, \pi),(\pi, 0)$ subbands in the configurations with three $f$ particles (fifth row).

Finally, we plot the (artificially broadened) spectral function of the isolated $2 \times 2$ cluster at $\beta=2$ in the fourth column of Fig. 8. The spectral peaks of the isolated plaquette can be clearly associated with the $K$-resolved spectral features 
of the embedded plaquette, although the latter are of course broadened due to lattice effects.

\section{CONCLUSIONS AND OUTLOOK}

We have simulated interaction ramps in the 2D FalicovKimball model using a nonequilibrium implementation of the DCA, and we compared the result for different clusters with up to eight sites. While these clusters are still too small to demonstrate a proper convergence of local and nonlocal expectation values with cluster size, we have shown that by averaging over different patch layouts, one can at least observe a systematic trend with cluster size (increasing correlations with increasing cluster size).

After a ramp to stronger interactions, the $c$ particles move away from the $f$ sites, which leads to strong nearestneighbor $c-f$ correlations in the nonthermal steady state. These enhanced correlations are, however, a manifestation of the trapping in a nonthermal state, since the thermal system with the same energy would have a more even distribution of $f$ particles and correspondingly weaker correlations. The opposite is true for the double occupancy, where the thermal state would exhibit a stronger reduction than the nonthermal steady state. By mapping out the differences between trapped and thermal expectation values for a range of initial and final interactions, we found that for ramps within the metallic or insulating regime, the deviations from thermal behavior are relatively small, while ramps across $U_{c}$ can lead to large deviations between the nonthermal steady-state value and the thermal reference.

Even in cases in which the local or nearest-neighbor observables reach almost thermal values after the quench, the nonequilibrium energy distribution function can show large deviations from a thermal one. We defined an energydependent effective temperature from the ratio of the retarded and lesser Green's function, and we showed that even for quenches within the metal regime, there are considerable variations, especially near the edges of the subbands of the spectral function (associated with different $f$ particle occupations). Not even within the subbands is it possible to define a meaningful effective temperature, so that a description of the nonequilibrium steady state in terms of a few parameters ( $f$-particle occupations, effective temperatures, and effective chemical potentials) seems difficult.

In the future, it would be interesting to extend this study to larger clusters using a Monte Carlo sampling of the initial $f$-particle configuration. Since the storage requirement of the nonequilibrium Green's functions is large, an explicit summation over all configurations, as done in this work, is not possible for substantially larger clusters. With clusters of size $8 \times 8$ or larger, it would be possible to explore issues related to Anderson localization, since an interaction ramp from $U_{0}=0$ is equivalent to the switch-on of a disorder potential, and the Falicov-Kimball model has been shown to exhibit a rich phase diagram with an Anderson insulating phase near the Mott transition [32].

\section{ACKNOWLEDGMENTS}

We thank L. Boehnke, D. Golež, and H. Strand for helpful discussions. The calculations were performed on the
Beo04 cluster at the University of Fribourg. A.J.H. and P.W. acknowledge support from ERC starting Grant No. 278023. We used the GNU Parallel software in the production of this paper [33].

\section{APPENDIX A: SELF-ENERGY CALCULATION}

The impurity self-energy fulfills the Dyson equation:

$$
\mathbf{G}=\mathcal{G}+\mathbf{G} \Sigma \mathcal{G} .
$$

Additionally, we define a contour function $\mathbf{X}$ that fulfills the following similar equation, and is also diagonal in $\mathbf{K}$ :

$$
\mathbf{G}=\mathcal{G}+\mathcal{G X G}
$$

Comparison to Eq. (A1) yields

$$
\Sigma=\mathbf{G}^{-1} \mathcal{G} \mathbf{X},
$$

whereas rearrangement yields

$$
\mathbb{1}+\mathbf{X G}=\mathcal{G}^{-1} \mathbf{G} .
$$

The combination of the last two equations produces a contour Fredholm equation of the second kind for the self-energy:

$$
(\mathbb{1}+\mathbf{X G}) \Sigma=\mathbf{X} .
$$

We still need to derive explicit forms for $\mathbf{X}$ and $\mathbf{X G}$. To that end, we insert the cluster solution Eqs. (14) and (17) into the Dyson equation (A1), which yields

$$
\mathbf{G}=\mathcal{G}+\mathcal{G}\left(\mathbb{1}+\sum_{\alpha} w_{\alpha} \mathbf{U}_{\alpha} \mathbf{R}_{\alpha}\right) \Sigma \mathcal{G} .
$$

By comparison to Eqs. (A2) and (A5), we find

$$
\mathbf{X \mathcal { G }}=\sum_{\alpha} w_{\alpha} \mathbf{U}_{\alpha} \mathbf{R}_{\alpha},
$$

and by applying $\mathcal{G}^{-1}$ from the right and using Eq. (17) we find

$$
\mathbf{X}=\sum_{\alpha} w_{\alpha} \mathbf{U}_{\alpha} \mathbf{R}_{\alpha} \mathbf{U}_{\alpha}
$$

\section{APPENDIX B: GENERALIZED GIBBS ENSEMBLE FOR THE FALICOV-KIMBALL MODEL}

Let us assume a Hamiltonian that can be written as a sum of conserved quantities, $\hat{I}_{\alpha}$, that commute with each other,

$$
H=\sum_{\alpha} \epsilon_{\alpha} \hat{I}_{\alpha}, \quad\left[\hat{I}_{\alpha}, \hat{I}_{\beta}\right]=0 .
$$

Consequently, all the $\hat{I}_{\alpha}$ commute with $\hat{H}$,

$$
\left[H, \hat{I}_{\alpha}\right]=0 \text {. }
$$

In this situation, the generalized Gibbs ensemble (GGE) is given by the density matrix

$$
\rho_{\mathrm{GGE}}=\frac{1}{Z_{\mathrm{GGE}}} e^{-\sum_{\alpha} \lambda_{\alpha} \hat{I}_{\alpha}},
$$

where

$$
Z_{\mathrm{GGE}}=\operatorname{Tr}\left[e^{-\sum_{\alpha} \lambda_{\alpha} \hat{I}_{\alpha}}\right]
$$


is the partition function for the GGE, and $\lambda_{\alpha}$ are Lagrange multipliers. If the system approaches the GGE in the longtime limit, then the $\lambda_{\alpha}$ are determined by the following set of conditions:

$$
\left\langle\hat{I}_{\alpha}\right\rangle_{\mathrm{GGE}}=\left\langle\hat{I}_{\alpha}\right\rangle_{t=+0}
$$

since each $\hat{I}_{\alpha}$ is conserved during the time evolution.

\section{Falicov-Kimball model}

In the case of the Falicov-Kimball model, the Hamiltonian is given by

$$
H=-t \sum_{\langle i, j\rangle}\left(c_{i}^{\dagger} c_{j}+\text { H.c. }\right)+U \sum_{i} \hat{n}_{i}^{f} \hat{n}_{i}^{c}-\mu_{c} \sum_{i} \hat{n}_{i}^{c} .
$$

Here $\hat{n}_{i}^{f}$ is conserved for each $i$,

$$
\left[H, \hat{n}_{i}^{f}\right]=0 .
$$

This allows one to simultaneously diagonalize $H$ and $\hat{n}_{i}^{f}$. In this basis, we can block-diagonalize the Hamiltonian in the form of (B1) as

$$
\begin{aligned}
& H=\sum_{\boldsymbol{n}_{f}} \hat{I}_{\boldsymbol{n}_{f}}-\mu_{c} \sum_{\boldsymbol{n}_{f}} \hat{N}_{\boldsymbol{n}_{f}}, \\
& \hat{I}_{\boldsymbol{n}_{f}}=\mathcal{P}_{\boldsymbol{n}_{f}}\left(-t \sum_{\langle i, j\rangle}\left(c_{i}^{\dagger} c_{j}+\text { H.c. }\right)+U \sum_{i} n_{i}^{f} \hat{n}_{i}^{c}\right) \text {, } \\
& \hat{N}_{\boldsymbol{n}_{f}}=\mathcal{P}_{\boldsymbol{n}_{f}} \sum_{i} \hat{n}_{i}^{c},
\end{aligned}
$$

where

$$
\sum_{\boldsymbol{n}_{f}}=\sum_{n_{1}^{f}=0,1} \sum_{n_{2}^{f}=0,1} \cdots \sum_{n_{N}^{f}=0,1}
$$

( $N$ is the number of lattice sites), and

$$
\mathcal{P}_{\boldsymbol{n}_{f}}=\left|n_{1}^{f}, n_{2}^{f}, \ldots\right\rangle\left\langle n_{1}^{f}, n_{2}^{f}, \ldots\right|
$$

is a projection operator onto the eigenspace of $\boldsymbol{n}_{f}=\left\{\hat{n}_{i}^{f}\right\}$. It is easy to see that

$$
\begin{gathered}
{\left[\hat{I}_{\boldsymbol{n}_{f}}, \hat{I}_{\boldsymbol{n}_{f}^{\prime}}\right]=0,} \\
{\left[\hat{I}_{\boldsymbol{n}_{f}}, \hat{N}_{\boldsymbol{n}_{f}^{\prime}}\right]=0,} \\
{\left[\hat{N}_{\boldsymbol{n}_{f}}, \hat{N}_{\boldsymbol{n}_{f}^{\prime}}\right]=0,}
\end{gathered}
$$

since

$$
\mathcal{P}_{\boldsymbol{n}_{f}} \mathcal{P}_{\boldsymbol{n}_{f}^{\prime}}=0 \quad\left(\boldsymbol{n}_{f} \neq \boldsymbol{n}_{f}^{\prime}\right)
$$

\section{Interaction quench}

Let us consider a situation in which the interaction parameter $U$ is quenched as $U=U_{-} \rightarrow U_{+}$at $t=0$. Correspondingly, we define

$$
\hat{I}_{\boldsymbol{n}_{f}}^{ \pm}=\mathcal{P}_{\boldsymbol{n}_{f}}\left(-t \sum_{\langle i, j\rangle}\left(c_{i}^{\dagger} c_{j}+\text { H.c. }\right)+U_{ \pm} \sum_{i} n_{i}^{f} \hat{n}_{i}^{c}\right) .
$$

Since $\hat{I}_{\boldsymbol{n}_{f}}^{ \pm}$and $\hat{N}_{\boldsymbol{n}_{f}}$ are quadratic in the fermionic operators and commute with each other, we can further diagonalize them with single-particle eigenstates $\left|\boldsymbol{n}_{f}, \alpha_{ \pm}\right\rangle$and eigenvalues $\varepsilon_{\boldsymbol{n}_{f}, \alpha_{ \pm}}$, where $\alpha_{ \pm}$labels each eigenstate before and after the quench. In this basis, we can write

$$
\begin{gathered}
\hat{I}_{\boldsymbol{n}_{f}}^{ \pm}=\mathcal{P}_{\boldsymbol{n}_{f}} \sum_{\alpha_{ \pm}} \varepsilon_{\boldsymbol{n}_{f}, \alpha_{ \pm}} \hat{n}_{\alpha_{ \pm}}^{c}, \\
\hat{N}_{\boldsymbol{n}_{f}}=\mathcal{P}_{\boldsymbol{n}_{f}} \sum_{\alpha_{ \pm}} \hat{n}_{\alpha_{ \pm}}^{c},
\end{gathered}
$$

where $\hat{n}_{\alpha_{ \pm}}^{c}=c_{\alpha_{ \pm}}^{\dagger} c_{\alpha_{ \pm}}$. We can see that

$$
\hat{N}_{\boldsymbol{n}_{f}, \alpha_{+}}=\mathcal{P}_{\boldsymbol{n}_{f}} \hat{n}_{\alpha_{+}}^{c}
$$

is conserved for each $\boldsymbol{n}_{f}, \alpha_{+}$after the quench. $\hat{N}_{\boldsymbol{n}_{f}, \alpha_{+}}$are the finest conserved quantities. $\hat{I}_{\boldsymbol{n}_{f}}^{+}$is linearly dependent on them $\left(\hat{I}_{\boldsymbol{n}_{f}}^{+}=\sum_{\alpha_{+}} \varepsilon_{\boldsymbol{n}_{f}, \alpha_{+}} \hat{N}_{\boldsymbol{n}_{f}, \alpha_{+}}\right)$.

The most general GGE is

$$
\rho_{\mathrm{GGE}}=\frac{1}{Z_{\mathrm{GGE}}} e^{-\sum_{\boldsymbol{n}_{f}, \alpha_{+}} \lambda_{\boldsymbol{n}_{f}, \alpha_{+}} \hat{N}_{\boldsymbol{n}_{f}, \alpha_{+}}},
$$

where $\lambda_{n_{f}, \alpha_{+}}$is the Lagrange multiplier. The constraint on it is given by Eq. (B5), which reads in the present case

$$
\left\langle\hat{N}_{\boldsymbol{n}_{f}, \alpha_{+}}\right\rangle_{\mathrm{GGE}}=\left\langle\hat{N}_{\boldsymbol{n}_{f}, \alpha_{+}}\right\rangle_{t=+0} .
$$

One can calculate both sides explicitly as

$$
\frac{1}{e^{\lambda_{n_{f}, \alpha_{+}}}+1}=\sum_{\alpha_{-}}\left|\left\langle\boldsymbol{n}_{f}, \alpha_{+} \mid \boldsymbol{n}_{f}, \alpha_{-}\right\rangle\right|^{2} f\left(\varepsilon_{\boldsymbol{n}_{f}, \alpha_{-}}\right),
$$

where $f(\varepsilon)=1 /\left(e^{\beta(\epsilon-\mu)}+1\right)$ is the initial thermal Fermi distribution. This completely determines $\lambda_{\boldsymbol{n}_{f}, \alpha_{+}}$.
[1] J. K. Freericks, V. M. Turkowski, and V. Zlatić, Phys. Rev. Lett. 97, 266408 (2006).

[2] H. Aoki, N. Tsuji, M. Eckstein, M. Kollar, T. Oka, and P. Werner, Rev. Mod. Phys. 86, 779 (2014).

[3] M. Moeckel and S. Kehrein, Phys. Rev. Lett. 100, 175702 (2008).

[4] M. Eckstein, A. Hackl, S. Kehrein, M. Kollar, M. Moeckel, P. Werner, and F. A. Wolf, Eur. Phys. J. Spec. Top. 180, 217 (2010).
[5] M. Eckstein, M. Kollar, and P. Werner, Phys. Rev. Lett. 103, 056403 (2009).

[6] J. Berges, S. Borsányi, and C. Wetterich, Phys. Rev. Lett. 93, 142002 (2004).

[7] N. Tsuji and P. Werner, Phys. Rev. B 88, 165115 (2013).

[8] N. Tsuji, M. Eckstein, and P. Werner, Phys. Rev. Lett. 110, 136404 (2013).

[9] P. Werner, N. Tsuji, and M. Eckstein, Phys. Rev. B 86, 205101 (2012). 
[10] M. Heyl, A. Polkovnikov, and S. Kehrein, Phys. Rev. Lett. 110, 135704 (2013).

[11] E. Canovi, P. Werner, and M. Eckstein, Phys. Rev. Lett. 113, 265702 (2014).

[12] N. Tsuji, P. Barmettler, H. Aoki, and P. Werner, Phys. Rev. B 90, 075117 (2014).

[13] M. Eckstein and P. Werner, Sci. Rep. 6, 21235 (2016).

[14] H. Keiter and J. C. Kimball, J. Appl. Phys. 42, 1460 (1971).

[15] P. Werner, E. Gull, O. Parcollet, and A. J. Millis, Phys. Rev. B 80, 045120 (2009).

[16] F. A. Wolf, I. P. McCulloch, and U. Schollwöck, Phys. Rev. B 90, 235131 (2014).

[17] K. Balzer, F. A. Wolf, I. P. McCulloch, P. Werner, and M. Eckstein, Phys. Rev. X 5, 031039 (2015).

[18] L. M. Falicov and J. C. Kimball, Phys. Rev. Lett. 22, 997 (1969).

[19] J. K. Freericks and V. Zlatić, Rev. Mod. Phys. 75, 1333 (2003).

[20] M. Eckstein and M. Kollar, Phys. Rev. Lett. 100, 120404 (2008).
[21] M. Rigol, V. Dunjko, V. Yurovsky, and M. Olshanii, Phys. Rev. Lett. 98, 050405 (2007).

[22] J. Hubbard, Proc. R. Soc. London, Ser. A 276, 238 (1963).

[23] U. Brandt and C. Mielsch, Z. Phys. B 75, 365 (1989).

[24] U. Brandt and C. Mielsch, Z. Phys. B 79, 295 (1990).

[25] U. Brandt and C. Mielsch, Z. Phys. B 82, 37 (1991).

[26] W. Metzner and D. Vollhardt, Phys. Rev. Lett. 62, 324 (1989).

[27] M. H. Hettler, A. N. Tahvildar-Zadeh, M. Jarrell, T. Pruschke, and H. R. Krishnamurthy, Phys. Rev. B 58, R7475 (1998).

[28] M. H. Hettler, M. Mukherjee, M. Jarrell, and H. R. Krishnamurthy, Phys. Rev. B 61, 12739 (2000).

[29] J. K. Freericks, Phys. Rev. B 77, 075109 (2008).

[30] N. Tsuji, T. Oka, and H. Aoki, Phys. Rev. Lett. 103, 047403 (2009).

[31] T. Maier, M. Jarrell, T. Pruschke, and M. H. Hettler, Rev. Mod. Phys. 77, 1027 (2005).

[32] A. E. Antipov, Y. Javanmard, P. Ribeiro, and S. Kirchner, Phys. Rev. Lett. 117, 146601 (2016).

[33] O. Tange, ;login: USENIX Mag. 36, 42 (2011). 